“C 2018 IEEE. Personal use of this material is permitted. Permission from IEEE must be obtained for all other uses, in any current or future media, including reprinting/republishing this material for advertising or promotional purposes, creating new collective works, for resale or redistribution to servers or lists, or reuse of any copyrighted component of this work in other works

Lattarulo, Ray, Daniel He, and Joshue Perez. "A Linear Model Predictive Planning Appro ach for Overtaking Manoeuvres Under Possible Collision Circumstances." 2018 IEEE Int elligent Vehicles Symposium (IV) (June 2018). doi:10.1109/ivs.2018.8500542. 


\title{
A Linear Model Predictive Planning Approach for Overtaking Manoeuvres Under Possible Collision Circumstances
}

\author{
Ray Lattarulo ${ }^{1}$, Daniel Heß ${ }^{2}$ and Joshue Pérez ${ }^{1}$
}

\begin{abstract}
Overtaking is one of the most difficult tasks during driving. This manoeuvre demands good skills to accomplish it correctly. In the overtaking considering multiple vehicles (more than a couple) is necessary to understand, predict and coordinate future actions of the other participants. These reasons make it a significant scenario for testing in the connected and automated driving field, with the main goal of predicting safe future states. In this sense, this work presents an overtaking method based on a linear Model Predictive Control (MPC) approach, which considers multiple participants involved in the scenario. This method adapts dynamically the trajectory for the manoeuvre in case of unexpected situations. Some of these changes consider other vehicles coming on the opposite lane or variations on participants' driving decisions. Additionally, the system considers passengers' comfort, the vehicle physical constraints and lateral actions of the vehicle decoupled of the longitudinal ones to simplify the problem.
\end{abstract}

\section{Introduction}

The last couple of decades have observed a great amount of progress in the field of Advanced DriverAssistance Systems (ADAS). Those have been boosted for a considerable quantity of challenges, projects and initiatives from public and private sectors. The common aim of most of them is to improve people's mobility, progressively demanded compared to previous periods [1].

Some of these improvements, addressing people mobility, have been done in the direction of: increasing passengers' comfort and safety while reducing travelling time. In the case of vehicular safety must be reached high level (difficult) requirements and standards. In the past, these standards have been satisfied using passive systems such as seat-belts and airbags, but currently, some ADAS systems can be chosen to reach fail operational level of automotive standards [2]. Some examples are driving conditions detection, potential hazardous situation detection, automated lighting, automated braking, lane departure warnings, blind spot detection, etc [3].

As a natural evolution of the ADAS, automated driving is gaining more interest in automakers and vehicle users, incorporating the concepts of complete automated control and cooperation during the driving process [4]. Even if the concept of automated driving goes back to 1980s with the demonstrations of Carnegie Mellon

\footnotetext{
${ }^{1}$ Tecnalia Research and Innovation, 48160, Derio, Vizcaya, Spain. \{rayalejandro.lattarulo; joshue.perez\}@tecnalia.com

${ }^{2}$ Deutsches Zentrum für Luft-und Raumfahrt, Lilienthalpl. 7, 38108 Braunschweig, Germany. daniel.hess@dlr.com
}

University with their NavLab vehicles, the attention increased with the demonstrations of the DARPA challenge in 2004, 2005 and 2007 [5].

In all these years, some of the advances developed on automated driving have been related with vehicle lateral and longitudinal controllers [6], management of communication of vehicles and infrastructure [7], path planning for defined environments and static obstacles [8], and some challenging manoeuvres, like overtaking, under controlled and specific scenarios [9]. The overtaking scenario is the main goal of this work, using connected vehicles to achieve more safety during the manoeuvre.

Our approach considers the problem stated on figure 1 , that is the existence of a collision if the trajectory planned does not consider the upcoming vehicle and variations on the participants' future decisions at any time. It is proposed a MPC solution to resolve the problem considering a lineal model (point-mass) and constrains related to passengers' comfort, safety (prevention of frontal and backward collisions) and vehicle dynamics. Additionally, it is considered that the information of position, speed, acceleration and angles, of the other participants, are broadcast by V2X (simplifying the task of modelling participants' future states).

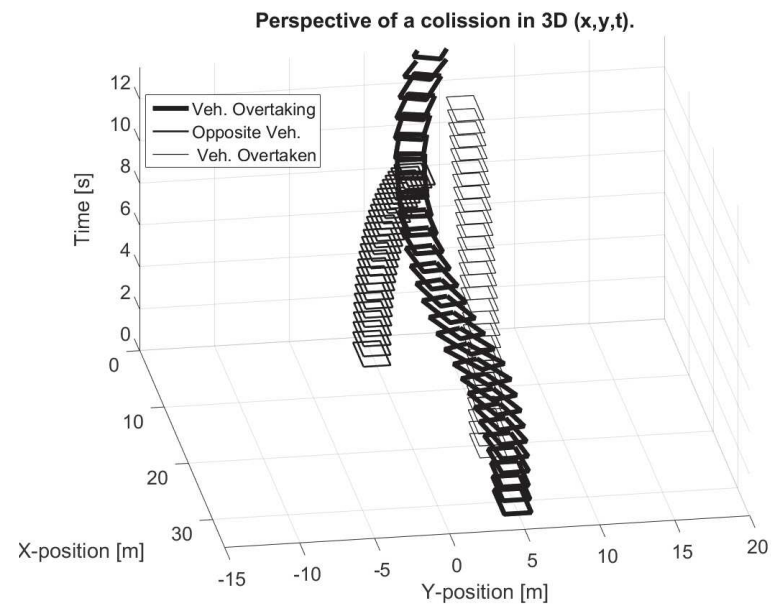

Fig. 1: Collision XY plane vs time.

The current work will be organized as follows: section II presents a brief review of the automated overtaking manoeuvre and the approaches made considering safety. Section III explains the platform used for integrating 
and testing the overtaking approach. In section IV is explained the Model Predictive Planning overtaking method for multiple actors achieved in this work. In section $\mathrm{V}$ is included some of the scenarios and test cases executed on a high fidelity simulator (highly precise dynamic vehicular modelling); and finally, the conclusions and future works are explained.

\section{Basis of automated overtaking}

Overtaking is one of the most challenging and hazardous manoeuvres on the driving process. It decreases the time of moving from one location to other, which sometimes is affected by the impact of vehicles at low speeds (traffic jam), and has a direct positive impact in the comfort of the passengers, keeping the desire speed [10]. Hence, overtaking helps to arrive to the destination faster, but increasing risks.

The work of [11] shows that risky situations under driving process are directly linked with higher possibilities to be involved in an accident, as it is the case for lane changes and overtaking. The percentage of accidents produced during the overtaking is between 4 and $10 \%$, where the main factor is faulty decisions taken while making the manoeuvre [12], [13].

Overtaking, classically, is separated in 3 different stages: i) A first lane change to the opposite side of the road, ii) The overtaking process and iii) A returning to the original lane [10]. This separation permits to solve the problem easier than just one single movement, as it is done in mobile robots with fixed obstacles.

In [14] a novel method to deal with the decision making process under overtaking has been presented. It has been based on Mixed Observability Markov Decision Process (MOMDP) which is a variant between a Markov Decision Process (MDP) and a Partly Observable Markov Decision Process (POMDP). The MOMDP is presented as a closer method, to the vehicle dynamics reality than the classical MDP; and it has less computation time than the POMDP. The work considered the information coming from communications V2V or perception (cameras, LiDAR and Radars) for the motion planning strategy. It is relevant to notice that the author highlights that the application uses offline tables to compute the solution of the problem. The computational complexity makes it non-viable for real-time implementations with current processors.

In [15] was stated a Rapidly-Exploring Random Tree (RRT) algorithm to avoid obstacles. To accomplish the task, a model of the vehicle and the control loop were needed to be included in the planner. The amount of time needed to achieve the task as well as the power of calculation were considered large.

Some other authors have used techniques based on intelligent control. Using fuzzy logic to control the steering wheel under overtaking manoeuvre, as well as obstacle avoidance process [16], [17]
III. Integration on automated vehicle control architecture

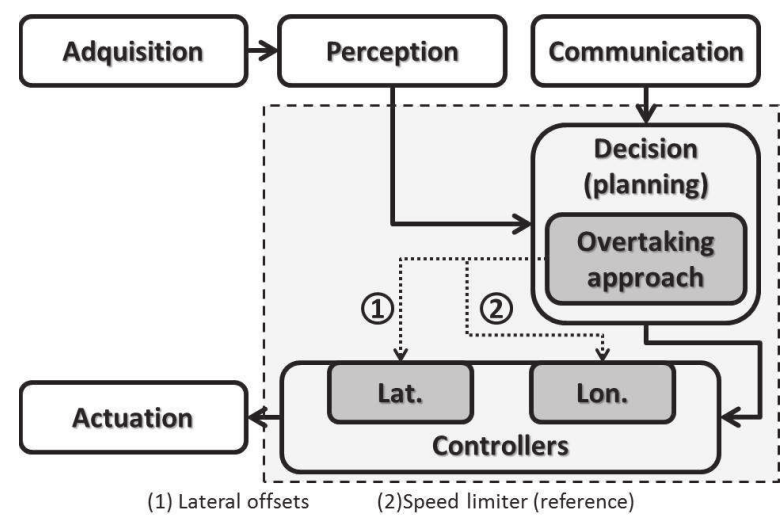

Fig. 2: System architecture considering overtaking.

In the current section, the control architecture used for implementing the overtaking manoeuvre will be presented (based on [18]), including how the MPC is integrated in it. Hence, it will be separated in two subsections: i) the general explanation of the architecture and its deployment based on modules, and ii) the integration of the algorithm, giving details of which modules are involved.

\section{A. Control architecture}

The control architecture used as base for the current work is the one presented on [18] and it is described in figure 2. It is a modular architecture, which integrates the capabilities of highly precise simulations (dynamically precise vehicle model). This architecture permits the validation of cooperative manoeuvres using other virtual participants with the simulation or the real automated vehicle.

The control architecture contains the six main blocks in automated vehicles [19], i.e. Acquisition, perception, communication, decision, control and actuation. The acquisition module is used for data collection of the virtual or real sensors and the vehicle odometry. These signals, which are measured not necessarily in physical values, are translated; making them comprehensive for logging and use in the next modules. The perception will generate the detailed description of the vehicle, and its surroundings, using the information coming from acquisition.

The main modules involved in the cooperative overtaking manoeuvre will be the communication, decision and control. The first module will be responsible of transmitting and receiving CAM messages, which contain information about position, heading angle, speed and acceleration, to be used in the MPC algorithm. The decision module will generate trajectories that the vehicle will follow and additionally, the approach presented in this work is part of this module. The control part will be divided into the lateral control that is the steering 
wheel control and the longitudinal control (throttle and braking systems).

Lastly, the actuation module consists of the low level control on the real platform and the assignments of position for steering wheel, throttle and braking on the simulated one.

\section{B. Integration on the automated driving framework}

To integrate the algorithm, the modules of decision and control were modified so as to support the MPC overtaking approach. The Model Predictive Overtaking approach has been integrated in the decision module (figure 2) to generate the reference changes in the lateral position and the reference speed during the overtaking, using the information obtained from the other participants. The module generates two signals for control that will be used with the lateral and angular error calculations to control the vehicle.

The two signals are shown on figure 2. The first one is the lateral error offset generated for the lane change process under an overtaking manoeuvre and the second one is the speed limitation for the speed reference. The last one is passed by a selector with the ideal speed of the path and, using as safety criteria, it is selected the lower speed (it is supposed that the ideal speed is the maximum of the road and it is decreased in case of a frontal obstacle).

\section{Proposed approach}

This work presents a planning method for the overtaking manoeuvre considering n-participants (in the test will be used 3 vehicles) in the scenario. The approach takes into account the following premises:

1) N-participants are considered for the trajectory generation.

2) The current ego information of each vehicle will be obtained with V2X, and the transmitted information will contain: position, heading angle, speed, acceleration, and the width and length for the vehicular physical description.

3) The manoeuvre must fit comfort criteria and physical limitations of vehicles.

4) The planned trajectory must be finished safely or a safe state will be reached.

5) At least two lanes are considered on the path and the right side will be at ego vehicle direction, but nonetheless the left side is in the opposite direction (in case of one lane, the approach cannot be executed).

To accomplish these premises, a linear model predictive control (Linear MPC) method is presented. It models the vehicle as decoupled lateral (steering wheel) and longitudinal (throttle and braking systems) actions to simplify the problem and decreasing computation time.

All the time, it is solved a possible lane change, but in case of a free lane it is not applied the manoeuvre. For this task, the system considers future predictions of

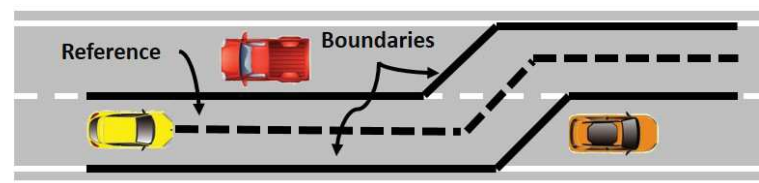

(a) Overtaking without any blocking.

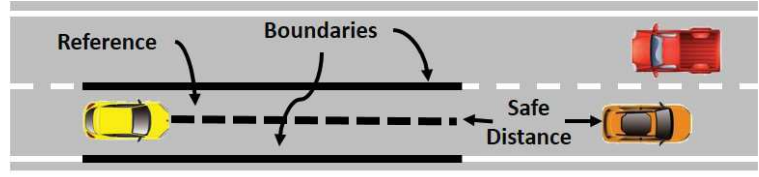

(b) Overtaking with vehicles blocking.

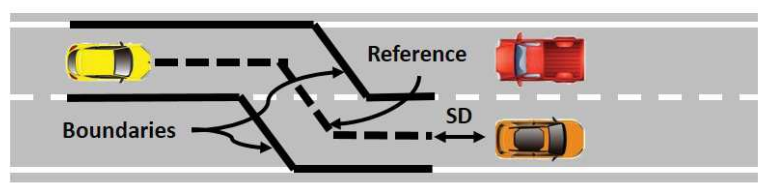

(c) Started overtaking and frontal vehicle

Fig. 3: Boundaries and reference in different situations

the ego vehicle (results obtained from past iteration) and calculates other participants' future predictions. When obtaining a positive result (considered as no collision), for the right lane, the lane will be kept. When there is any participant blocking the lane (for the moment of time of the prediction), then a lane change will be applied. In the case of a no crashing result (positive), the lane change will be done; whereas in the case of a crash prediction, as it is shown in figure 1 the system will return the vehicle to its safe lane (right side), introducing speed reduction, avoiding the frontal crash.

The crash prediction is obtained using vehicle future MPC predictions (in the ego vehicle) as well as the projection of future positions for the other participants (using motion equations). By using the information of the width and length of each vehicle is verified that the vehicles do not collide in future time steps.

Once it is known the possible future conditions of the vehicle under an overtaking situation, the boundaries and the reference will be set based on the information obtained in the past iteration. In the figure 3a is shown the case of having a free left lane and a blocked right lane, where the boundaries are set in terms of being into the lane considering the vehicle width (containing completely the vehicle into the lane) and the reference is located in the middle of the boundaries. In the case of figure $3 \mathrm{~b}$ is shown the consideration that is not possible to do the overtaking and the right lane is blocked by a vehicle. The boundaries are left into the right lane but the maximum distance is limited to a maximum distance that permits keeping a safe distance between the ego vehicle and the frontal one. Finally figure $3 \mathrm{c}$ shows that the boundaries are set to return to the original lane because there is a blocking in the left lane. The total distance is constrained 
to avoid a collision with the frontal vehicle and keeping a safe distance.

The longitudinal model used for the approach is based on a triple integrator chain, that will provide speed set points to a closed loop controller. The control loop will correct the error obtained for the relative precision of the model for a dynamically complex system, as a vehicle. The model is presented in the equation 1 :

$$
d_{l o n}=\iiint J_{l o n}(t) d t d t d t
$$

The model is a linear differential equation system, where the involved state variables are longitudinal distance, speed and acceleration with jerk as control input. This gives as a result the following state space representation:

$$
\left[\begin{array}{l}
\dot{d}_{l o n} \\
\dot{v}_{l o n} \\
\dot{a}_{l o n}
\end{array}\right]=\left[\begin{array}{lll}
0 & 1 & 0 \\
0 & 0 & 1 \\
0 & 0 & 0
\end{array}\right]\left[\begin{array}{l}
d_{l o n} \\
v_{l o n} \\
a_{l o n}
\end{array}\right]+\left[\begin{array}{l}
0 \\
0 \\
1
\end{array}\right] J_{l o n}
$$

The variable to be regulated is the speed error and it will be set as a Quadratic Problem (QP) optimizing jerk. The distance is used as a constraint variable to prevent the frontal collision. Acceleration is used as another constraint variable to set the maximum acceleration that the system can achieve by design (max. acceleration and max. deceleration) and lastly, the jerk is used as a comfort parameter as it is mentioned in the work of [20]. These statements can be summarize in the following inequalities:

$$
\begin{aligned}
0 & \leq d_{\text {lon }} \leq D_{\text {veh }_{\text {front }}} \\
D e c_{\text {Max }} & \leq a_{\text {lon }} \leq A c c_{\text {Max }} \\
J_{\text {Min }_{\text {Comfort }}} & \leq J_{\text {lon }} \leq J_{\text {Max }}
\end{aligned}
$$

For the lateral domain, reference changes of the lateral offset to execute the lane change has been done using a double integrator chain. It is shown as follows:

$$
d_{l a t}=\iint a_{l a t}(t) d t d t
$$

Using the classical representation, the ODE is reduced to a state variable vector of lateral position offset and lateral speed (rate of change of offset) with lateral acceleration as control input:

$$
\left[\begin{array}{c}
\dot{d}_{\text {lat }} \\
\dot{v}_{\text {lat }}
\end{array}\right]=\left[\begin{array}{ll}
0 & 1 \\
0 & 0
\end{array}\right]\left[\begin{array}{l}
d_{\text {lat }} \\
v_{\text {lat }}
\end{array}\right]+\left[\begin{array}{l}
0 \\
1
\end{array}\right] a_{\text {lat }}
$$

The solution will be given using a QP formulation (getting the solution in all time steps), minimizing the difference between the lateral tracking error (vehicle with the trajectory) and the lateral offset given to execute the manoeuvre (MPC approach). The rate of change on this system is used as a constrained variable for the correct tracking of the lateral controller. The lateral acceleration constraint is used as comfort parameters for passengers [21].

\section{Test cases}

The two use cases used to validate the algorithm are: i) 3 participants with enough space to finish completely the manoeuvre (it is not necessary to plan again the manoeuvre), and ii) 3 participants but the system will execute the planning again to avoid the collision during the manoeuvre (the trajectory is planned again). Both cases will be explained as follows.

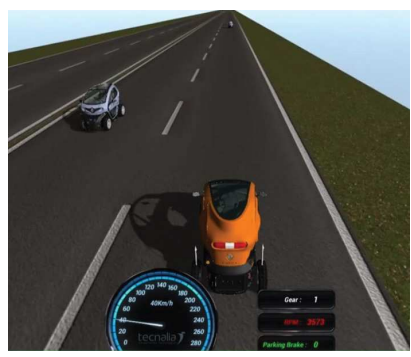

(a) Passing.

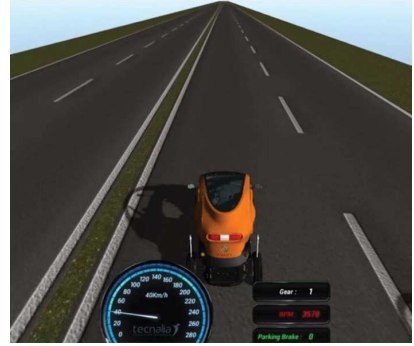

(c) Overtaking.

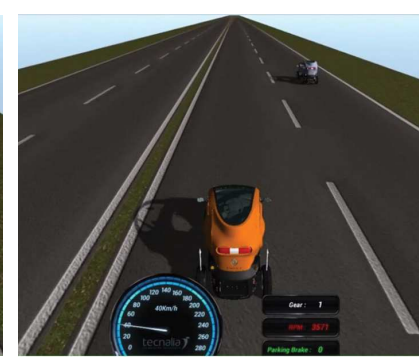

(b) Taking the other lane.

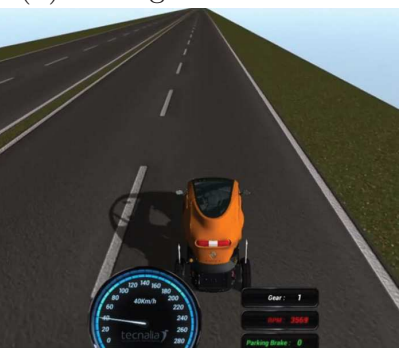

(d) Returning.

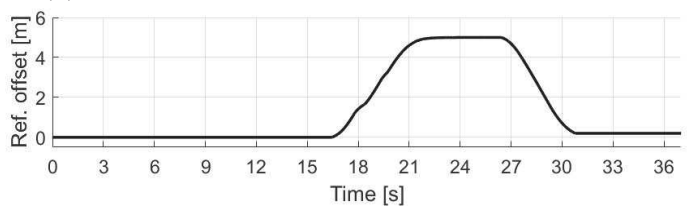

(e) Lateral offset.

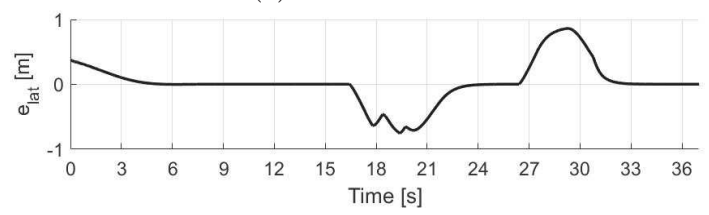

(f) Lateral error

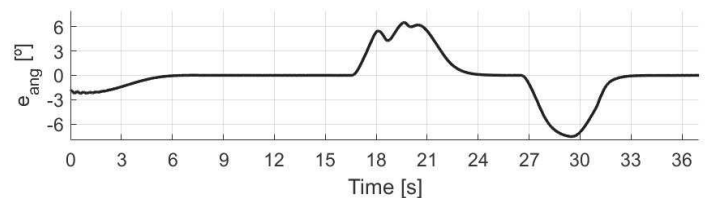

(g) Angular error.

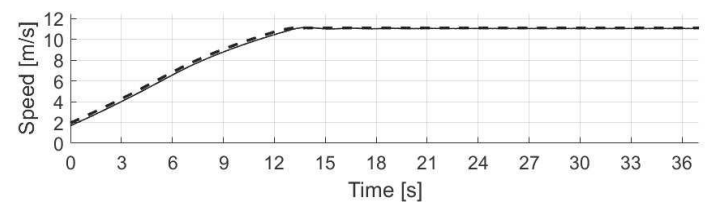

(h) Vehicle and reference speed.

Fig. 4: Results of test case 1. 


\section{A. Test case 1: Planning without adaptation}

This first scenario presents 3 vehicles, where the first is the automated vehicle (overtaking vehicle), the second one (blocking vehicle) is the vehicle with low speed in front of the one doing the overtaking, and the third one is a vehicle moving in the opposite direction (opposite lane).

Figures $4 \mathrm{a}$ to $4 \mathrm{~d}$ shows the process of the automated overtaking on 4 different time instances: i) the third vehicle passing, ii) the first one taking the opposite lane, iii) the overtaking process and iv) the returning. As it is shown on that sequence the third vehicle did not affect the process of overtaking.

The information can be validated with the control variables where figure $4 \mathrm{e}$ shows the lateral offset planned for the overtaking, without any change on the intend of overtaking the vehicle, it continues the evolution from 0 to 5 meters of offset without any change. The value of 5 meters refers to the distance from the middle of the starting lane to the middle of the opposite lane.

Figures $4 \mathrm{f}$ and $4 \mathrm{~g}$ depict the correct following of the lateral reference, with a lateral error $e_{l a t}$ of approximately $0.75[\mathrm{~m}]$ for a period of time around 3 seconds. In other hand, the angular error $e_{\text {ang }}$ (heading tracking error) has presented a maximum deviation between the vehicle heading and the angle of the reference path of $6^{\circ}$ during the overtaking. Additionally, the smoothness on the evolution of both variables could be considered as comfort for the passengers.

Lastly, the speed remains constant after arriving at top speed, as the manoeuvre is not affected by the oncoming vehicle. This is, because the overtaking manoeuvre is not affected by the vehicle in front.

\section{B. Test case 2: Planning adapted dynamically}

This test case, same as previous one, uses 3 vehicles, where the first one is the one that is doing the overtaking the second one is the vehicle that blocks the first one and the third one is a vehicle in the opposite direction.

The sequence of pictures in figure 5a depict a 200 meter long road segment with two lanes, each 5 meters wide and one for each direction. They show the evolution of the overtaking experiment. In this test case, vehicle 1 is trying to execute an overtaking manoeuvre based on the information arriving from vehicle 2 and the distance towards vehicle 3 (figure $5 \mathrm{a}$ top part at 2 seconds). Vehicle 1 starts the overtaking manoeuvre, but the information send by vehicle 3 induces vehicle 1 to remain in its lane and to adapt the speed with respect to vehicle 2 (plots between 5 and 9 seconds). After this, vehicle 1 continues the overtaking process (from 9 to 13 seconds) until it starts the returning process (from 13 to 17 seconds), arriving to the original lane at the end (19 seconds).

Figure 5b shows the process of returning to the original lane cause by a blockage in the opposite lane made by the vehicle 3 . This part of the manoeuvre happens between 5

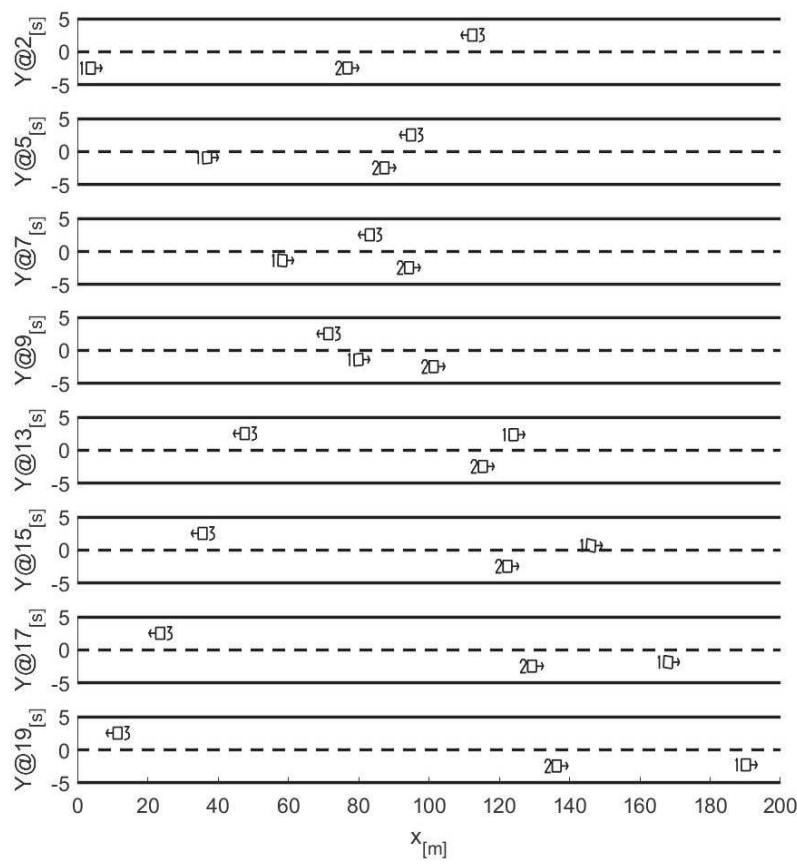

(a) Time secuence for overtaking in test case 2 .

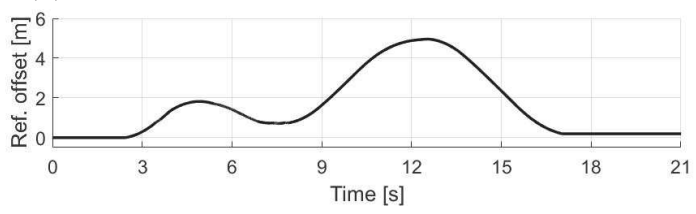

(b) Lateral offset.

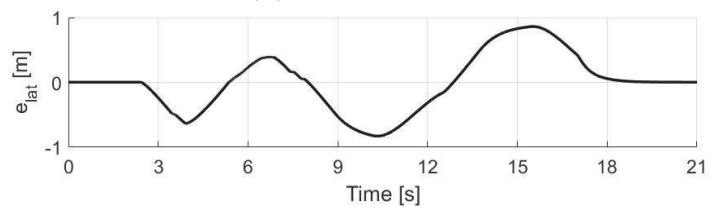

(c) Lateral error.

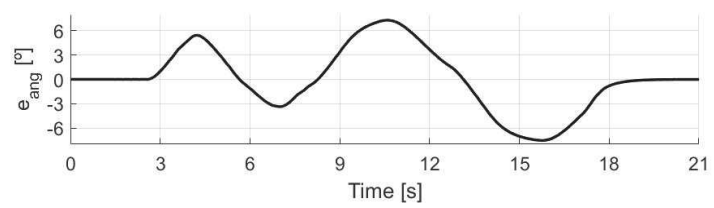

(d) Angular error.

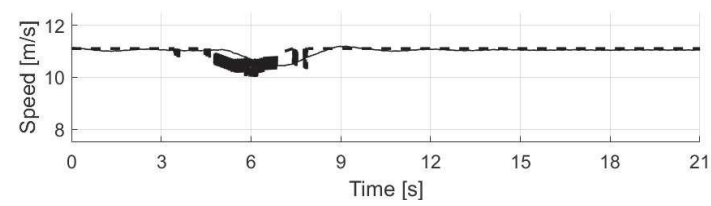

(e) Vehicle and reference speed.

Fig. 5: Results of test case 2.

and 9 seconds, and it shows a reduction on the offset from 2 meters to less that 0.75 meters, doing a safe correction avoiding a possible collision.

Figures $5 \mathrm{c}$ and $5 \mathrm{~d}$ show the tracking of the lateral controller, where the maximum lateral error $e_{\text {lat }}$ is under 
0.75 meters for the tracking of the reference position. The angular error $e_{a n g}$ is lower than 7 degrees with respect to the angle of the path. It is relevant to notice that for the type of control used in the experiments the lateral and angular errors can be improved (control tuning) but it is not the purpose of the current work.

Finally, figure 5e depicts the speed correction made by the system under the avoidance of the third vehicle for safety reasons. Vehicle 1 reduces the speed in order to adapt it to the speed of vehicle 2, avoiding a potential rear end.

\section{Conclusions}

In this work, a linear model predictive planning approach for overtaking manoeuvre, considering multiple participants conditions, has been presented. The approach has been implemented using a dual-modular automated driving control architecture (all the task are grouped and categorize based on their function). This approach is categorized as part of motion planning (decision module) and it demands a lateral control with a high level of robustness implemented in the architecture to handle with the correction of errors, which are caused by the difference between non-linear simulation model and linear model assumed during motion planning

The MPC approach permits to predicts possible collision among multiple vehicles involved in the overtaking, and re-calculating the speed and trajectory planned in case of modification on participants' states; the dynamic replanning will keep safe conditions during driving.

The future works related to the Model Predictive Planning Approach will consider the deployment on the real platform, testing the behaviour of the set point tracking on the lateral and longitudinal controllers during the manoeuvre. Lastly, user acceptance must be tested based on the indicators: comfort, safety and interaction with other participants in the manoeuvre.

\section{ACKNOWLEDGMENT}

The authors gratefully acknowledge financial support from the European Commission project UnCoVerCPS under grant number 643921

\section{References}

[1] E. Bertolazzi, F. Biral, and M. Da Lio. Future advanced driver assistance systems based on Optimal Control: the influence of "risk functions" on overall system behavior and on prediction of dangerous situations. In IEEE Intelligent Vehicles Symposium, 2004, pages 386-391, June 2004.

[2] H. Fritz, A. Gern, H. Schiemenz, and C. Bonnet. CHAUFFEUR Assistant: a driver assistance system for commercial vehicles based on fusion of advanced ACC and lane keeping. In IEEE Intelligent Vehicles Symposium, 2004, pages 495-500, June 2004.

[3] George Dimitrakopoulos. Current Technologies in Vehicular Communication. Chapter 4, Advanced Driver Assistance Systems (ADAS). Springer, 2017.

[4] George Dimitrakopoulos. Current Technologies in Vehicular Communication. Chapter 6, the future: Towards autonomous driving. Springer, 2017.
[5] Ömer Sahin Tas, Florian Kuhnt, J. Marius Zöllner, and Christoph Stiller. Functional system architectures towards fully automated driving. In 2016 IEEE Intelligent Vehicles Symposium (IV), pages 304-309, June 2016.

6] E. Onieva, J. E. Naranjo, V. Milanés, J. Alonso, R. García, and J. Pérez. Automatic lateral control for unmanned vehicles via genetic algorithms. Applied Soft Computing, 11(1):13031309, January 2011.

[7] V. Milanes, J. Villagra, J. Godoy, J. Simo, J. Perez, and E. Onieva. An Intelligent V2i-Based Traffic Management System. IEEE Transactions on Intelligent Transportation Systems, 13(1):49-58, March 2012.

[8] D. González, J. Pérez, R. Lattarulo, V. Milanés, and F. Nashashibi. Continuous curvature planning with obstacle avoidance capabilities in urban scenarios. In 17th International IEEE Conference on Intelligent Transportation Systems (ITSC), pages 1430-1435, October 2014.

[9] Ray Lattarulo, Mauricio Marcano, and Joshué Pérez. Overtaking maneuver for automated driving using virtual environments. In International Conference on Computer Aided Systems Theory (EuroCAST), pages 446 - 453. Springer, Cham, February 2017. DOI: 10.1007/978-3-319-74727-9_54.

[10] F. Wang, M. Yang, and R. Yang. Conflict probability estimation based overtaking for intelligent vehicles. IEEE Transactions on Intelligent Transportation Systems, 10(2):366-370, June 2009.

[11] Se Jin Park, Murali Subramaniyam, Seoung Eun Kim, Seunghee Hong, Joo Hyeong Lee, and Chan Min Jo. Older Driver's Physiological Response Under Risky Driving Conditions-Overtaking, Unprotected Left Turn. In Advances in Applied Digital Human Modeling and Simulation, Advances in Intelligent Systems and Computing, pages 107-114. Springer, Cham, 2017.

[12] Eleni I. Vlahogianni. Modeling duration of overtaking in two lane highways. Transportation Research Part F: Traffic Psychology and Behaviour, 20(Supplement C):135-146, September 2013 .

[13] T Mocsari. Analysis of the Overtaking Behaviour of Motor Vehicle Drivers, volume 2. Acta Technica Jourinensis, January 2009.

[14] Volkan Sezer. Intelligent decision making for overtaking maneuver using mixed observable Markov decision process. Journal of Intelligent Transportation Systems, 0(0):1-17, May 2017.

[15] Y. Kuwata, G. A. Fiore, J. Teo, E. Frazzoli, and J. P. How. Motion planning for urban driving using RRT. In 2008 IEEE/RSJ International Conference on Intelligent Robots and Systems, pages 1681-1686, September 2008.

[16] H. H. Chiang, Y. L. Chen, B. F. Wu, and T. T. Lee. Embedded Driver-Assistance System Using Multiple Sensors for Safe Overtaking Maneuver. IEEE Systems Journal, 8(3):681-698, September 2014.

[17] Vicente Milanés, David F. Llorca, Jorge Villagrá, Joshué Pérez, Carlos Fernández, Ignacio Parra, Carlos González, and Miguel A. Sotelo. Intelligent automatic overtaking system using vision for vehicle detection. Expert Systems with Applications, 39(3):3362-3373, February 2012.

[18] Ray Lattarulo, Joshué Pérez, and Martin Dendaluce. A complete framework for developing and testing automated driving controllers. IFAC-PapersOnLine, 50(1):258-263, July 2017.

[19] David González, Joshué Pérez, Vicente Milanés, and Fawzi Nashashibi. A review of motion planning techniques for automated vehicles. In Transactions on Intelligent Transportation Systems, pages 1135 - 1145. IEEE, April 2016. DOI: 10.1109/TITS.2015.2498841.

[20] Jorge Villagra, Vicente Milanés, Joshué Pérez, and Jorge Godoy. Smooth path and speed planning for an automated public transport vehicle. Robotics and Autonomous Systems, 60(2):252-265, February 2012.

[21] Ray Lattarulo, Enrique Martí, Mauricio Marcano, Jose Matute, and Joshué Pérez. A speed planner approach based on bézier curves using vehicle dynamic constrains and passengers comfort. In 50th International Symposium on Circuits and Systems (ISCAS). IEEE, 2018. 